\title{
The Nature of Heat and the Absolute Zero Temperature
}

\author{
Xingwu Xu \\ Research Institute of Hefei Gotion High-tech Power Energy Co., Ltd. 599 Daihe Road, Hefei, Anhui 230012, P. R. China \\ Email: xuxingwu@gotion.com.cn
}

Received Nov 2020

Received in revised: Dec 2020

Published: Dec 2020

\begin{abstract}
This paper starts with the most basic concept of heat as well as temperature, historically investigates the understanding of the nature of heat, the conclusion is that the nature of heat is just a form of energy. This energy includes the zero-point energy providing by the motion of all subatomic particles. The new definition of temperature should be that it is the degree of matter's motion. These matters include subatomic particles. Therefore, at the absolute zero, the "temperature" should still exist. On accounting of no subatomic particles' motion in the singularity of the black hole, I proved that there exists a new absolute zero temperature there, which is lower than the existing one. The theory proposed in this paper can be supported by following means: measuring the temperature inside the black hole, letting electrons stop moving, and designing a Casimir vacuum pump.
\end{abstract}

Keywords: Nature of Heat, Absolute Zero, Black Hole, Temperature.

C2020 The Authors. Published by Fundamental Journals. This is an open access article under the CC BY-NC https://creativecommons.org/licenses/by-nc/4.0/

https://doi.org/10.14331/ijfps.2020.330140

\section{INTRODUCTION}

What is heat? The original concept of heat came from the fire. However, for a long time, the humanity still doesn't know what heat is. In the 17 th century, Becher and Stahl introduced phlogiston to explain processes such as combustion and rusting. This theory considers that phlogiston is a fire element which contains within combustible substances and has a weight. During combustion process the phlogiston escapes out and combines with the air that surrounds it (Best, 2015), releasing heat and light. The phlogiston theory was overthrown by French chemist Lavoisier, because it cannot explain the phenomenon of some metals gained mass when they burned. Lavoisier considered the nature of heat as a "matter of heat" which is called "caloric" (Best, 2015). The "caloric theory" was queried by Count Rumford. In 1798, when superintending the boring of cannon, Rumford found that a very considerable degree of heat produces by friction. "A thorough investigation of them seemed even to bid fair to give a farther insight into the hidden nature of heat; and to enable us to form some reasonable conjectures respecting the existence or non- 
existence, of an igneous fluid" (Rumford, 1798). The Rumford's experimental results show that heat is connected with the mechanical motion-the friction.

In researching the efficiency of steam engine, Carnot found that heat can produce motion, and heat is also due to movement. However, Carnot considers that heat itself is not a kind of motion, it is still a caloric (Carnot, 1897).

Maxwell believes that heat is not a substance, the reason is that heat can be generated. Maxwell came to the conclusion that heat is a mode of motion, a form of energy. But "the motion must be carried on by parts of the body too minute to be seen separately" (Maxwell \& Pesic, 2001). "We have now arrived at the conception of a body as consisting of great many small parts, each of which is in motion. We shall call any one of these parts a molecule of the substance" (Maxwell \& Pesic, 2001).

About the internal fluid motion, Bernoulli put forward a kinetic theory, which considers that there is so-called "vis viva" stored in the compressed air (e.g., in a vessel) (Pacey \& Fisher, 1967). The pressure of air is due to the impact of its particles on the sides of the vessel containing it (Maxwell \& Pesic, 2001). The modern concept of heat is that heat is energy, heat is the microscopic motion of internal particles of the body, such as molecules, atoms, etc.

On the molecular motion level, the Third Law of Thermodynamics says that absolute zero is unattainable. However, there are still a lot of subatomic particles keeping motion even at the absolute zero temperature. For example, in the nuclear spin system the "negative absolute temperatures" can be attained (Purcell \& Pound, 1951). It is due to the inversion of the ratio of subatomic particles' population.

The motion of those subatomic particles is driven by the zeropoint energy field. This energy is called Zero Point Energy (ZPE). ZPE was proved by "Lamb shift" and "Casimir effect" etc. The existence of zero-point energy manifests that there is a large amount of energy at the temperature of absolute zero.

\section{THE ABSOLUTE ZERO TEMPERATURE IN THE SINGULARITY OF BLACK HOLE}

Here I will begin with the basic concept and ask the question: what is the temperature? According to our present knowledge, the temperature is a physical quantity expressing hot and cold. What does the "hot and cold" mean? This question brings us back to the problem of heat's nature. As mentioned above, heat is energy, heat is the microscopic motion of molecules or atoms.

There is a contradiction: does this energy include the microscopic motion of subatomic particles? If the answer is yes, at the absolute zero there is a zero-point energy, there exists the high-speed motion of those particles, so there must be the temperature. Therefore, the revised concept of temperature should be, Temperature is the degree of matter's motion.

According to Fischer et al. (2007), the temperature is proportional to the mean translational kinetic energy of molecules. If the molecules are replaced by subatomic particles, it is just the revised concept of temperature.

As we know, at the singularity of black hole the photon will stop moving. Naturally the question arise: what is the temperature in the singularity? Till now, the problem of initial singularity is still a cosmological puzzle (Medina, Nowakowski, \& Batic, 2019).

So does the singularity in the black hole. From the viewpoint of matter's motion, because the photons have stopped moving in the singularity of the black hole, the temperature will be new zero there. In fact, the temperature is related to kinetic energy of the oscillators,

$$
T \propto E_{k i n}=\frac{1}{2} h v_{o s c i}
$$

where $v_{\text {osci. }}$ is the frequency of the oscillator, $\mathrm{h}$ is the Planck constant. If the $v_{o s c i}=0$, the temperature at the singularity has to be a new zero: $T_{s}=0$. If our Earth became a black hole, the electron would stop moving. According to the calculation formula of Schwarzschild radius,

$$
R_{S}=\frac{2 G M}{c^{2}}
$$

where $G$ is the gravitational constant, $M$ is the mass of a star, $C$ is the speed of light. For the earth, the $R_{S} \approx 9 \times 10^{-3} \mathrm{~m}$. Now let's compare the ratios below: atomic radius: nuclear radius and Schwarzschild radius: the earth's radius. The atomic radius is around $10^{-10} \mathrm{~m}$ (Slater, 1964), nucleus radius: $10^{-15} \mathrm{~m}$ (Angeli \& Marinova, 2013) $R_{\text {earth }}=$ $12742000 \mathrm{~m}$, therefore

$$
\begin{gathered}
r_{\text {nucl }} / r_{\text {atom }}=10^{-5} \\
R_{s} / R_{\text {earth }} \approx 10^{-10}
\end{gathered}
$$

Obviously, not only the all electrons fall down into the nucleus, but also shrink by $10^{5}$ times. It means that all electrons stop moving in the inside of the black hole.

However, how do the electrons go to the singularity step by step and stop moving there at last? What is the change of temperature during this process? Here I will put forward a simple model. First of all, I just consider the first layer of atoms which are in the reacting surface of the black hole's formation (Fig. 1).

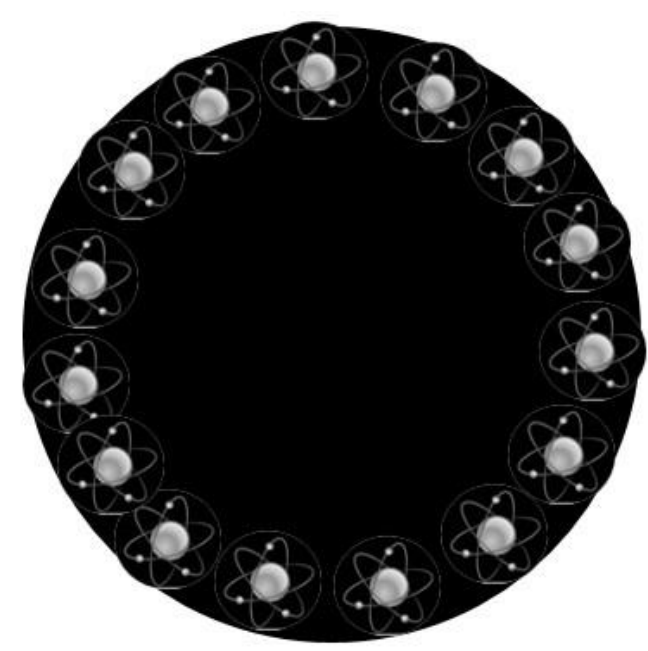

Fig. 1 The first layer of atoms on the surface of the event horizon of the black hole.

Suppose that there are many energy levels from the ground state to the nucleus (Fig.2). 


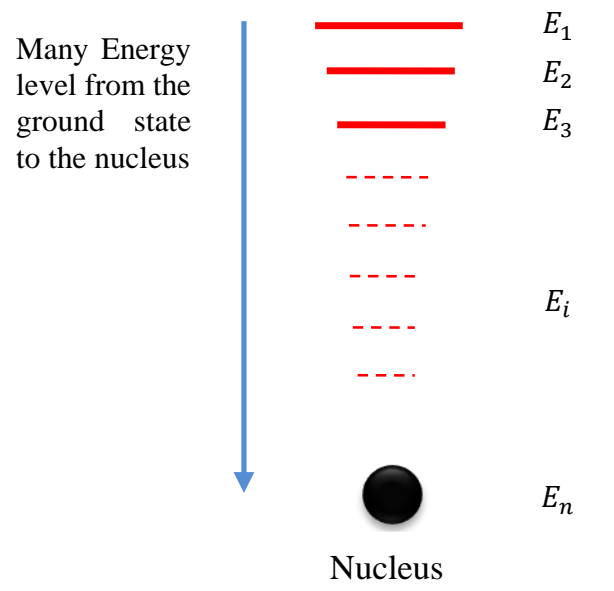

Fig. 2 Many energy levels from the energy level of ground state to the nucleus.

Here I will borrow the concept of quantum refrigerator, which was first put forwarded by (Scovil \& Schulz-DuBois, 1959). The main principle is as follows (Scovil \& Schulz-DuBois, 1959).

As any heat engine, this system should be reversible so that it acts as a refrigerator. This is indeed the case. Suppose a quantum $h v$ is applied to the signal transition. It causes an ion to go from state 1 to 2 . The ion may further jump to state 3 if the energy hv is supplied by the cold reservoir.

The cycle is finally completed when the ion returns from state 3 to state 1 while the energy hv is communicated to the hot reservoir. In this process, energy is extracted from the idler transition, which is from the cold reservoir, so that it is refrigerated. The system here is the quantum system of threelevel masers, as shown in Fig. 3 (Scovil \& Schulz-DuBois, 1959).

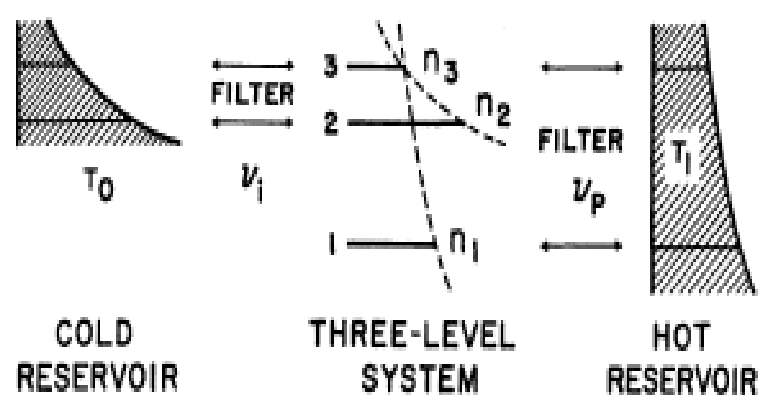

Fig 3. The three-level system in thermal contact with two heat reservoirs.

Similarly, if the electrons are forced from higher energy level to lower energy level, the energy will emit out, and the temperature will fall. According to (Quan, 2009), the surface stress $\mathrm{F}$ (which is same as the pressure $\mathrm{P}$ ) of a quantum gas system is:

$$
F=\sum_{n} P_{n} \frac{d E_{n}}{d L}
$$

Where $P_{n}$ is the occupation of probability in the n-th eigenstate, $E_{n}$ is the $n$-th eigenenergy of the quantum- mechanical system with the Hamiltonian $H=\sum_{n} E_{n}|n\rangle\langle n|$, $\mathrm{L}$ is the generalized coordinate corresponding to $F$. From Eq. (5) we have,

$$
\frac{d F}{d L}=\sum_{n} \frac{d P_{n}}{d L} \frac{d E_{n}}{d L}+\sum_{n} P_{n} \frac{d^{2} E_{n}}{d L^{2}}
$$

Let's back to the issue of the black hole. Here the generalized coordinate $\mathrm{L}$ will be replaced by polar coordinate $\mathrm{r}$. Assume that all atoms were in the "frozen" state in the black hole, then the energy levels are just the function of $r: E_{n}=k r$, thus we have,

$$
\frac{d^{2} E_{n}}{d r^{2}}=0
$$

The equation Chandrasekhar used becomes (Chandrasekhar, 1984).

$$
\sum_{n} \frac{d P_{n}}{d r} \frac{d E_{n}}{d r_{n}}=-\frac{G M(r)}{r^{2}} \rho
$$

Where $G$, is the gravitational constant, $M(r)$ is the mass interior to the sphere of the radius $r, \rho$ is the mass density of the black hole. Equation (8) means that the electrons are forced by strong gravity to jump from the higher energy level $E_{h}$ down into the lower energy level $E_{1}$, and emit the energy $h v$,

$$
E_{h}-E_{l}=h v
$$

This energy becomes the radiation and brings some amount of heat away. At the same time the system absorbs the heat from the black hole and the temperature is lowered. As Fowler put it, "A loss of energy by radiation means necessarily a loss of entropy, and therefore a fall of temperature." (Fowler, 1926). In this way, the atoms will be cooled layer by layer. And the temperature will be lower and lower.

When the process going to the surface of the event horizon, the temperature $T=h c^{3} / 8 \pi k G M$, it is around $10^{-7} \mathrm{k}$ for $M=$ $3 M_{\odot}$. As the temperature goes down, there must be a point where the temperature equals to absolute zero $\left(T_{k}=0^{K}\right)$. This process can be shown in Fig 4 . The temperature will be further lowered to its lowest temperature: the temperature of the singularity $T_{s}$.

According to the equation (1), $T_{s} \propto E_{\text {kin }}=\frac{1}{2} h v_{\text {osci. }}=0$, because in the inside of the black hole all motion (include subatomic particles such as Fermi gas, Bose gas, and electrons etc.) stops, it is the lowest temperature in the universe. Thus we have: $n_{2}$

$$
T_{s}<T_{k}
$$

Many atom layers from the surface of the event horizon to the center of the black hole. The singularity of the black hole. The temperature on the event horizon of the black hole is larger than absolute zero, the temperature at the singularity is zero $\left(T_{s}=0\right), T_{s}<T_{k}$. One might argue that it violates the third law of thermodynamics, the answer is no. Because in the environment of the earth or solar system, there are a large amount of subatomic particles (Dirac sea, vacuum field) pervading everywhere, which are in high-speed moving. 


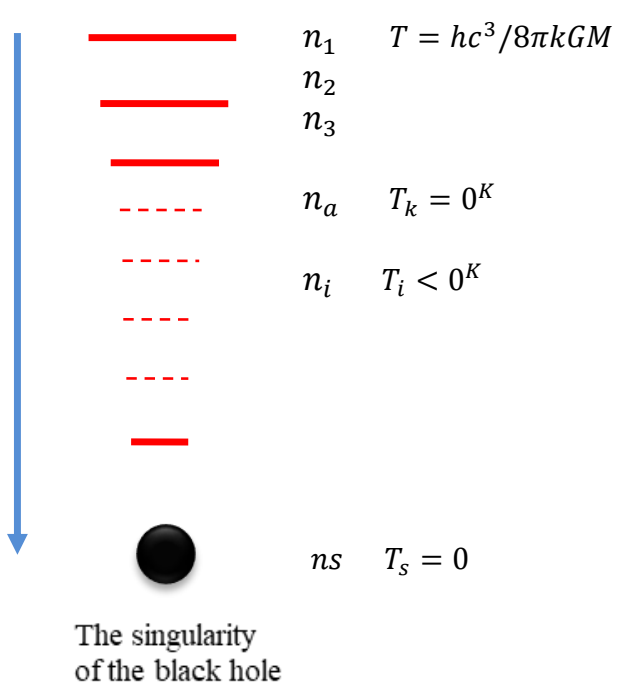

Fig 4. The atoms are cooled layer by layer. Many atom layers from the surface of the event horizon to the center of the black hole.

In this environment, the third law of thermodynamics is absolutely correct. The absolute zero is unattainable. However, for other environments in the universe where subatomic particles stop moving, or where there are no subatomic particles (the real void), there will be a new absolute zero there.

\section{PREDICTION AND PROOF}

The prediction of the theory mentioned above is that there exists the new absolute zero temperature (lower than available absolute zero) at the singularity of the black hole where all particles stop moving, or in the area of the real void of the universe. Some of these proofs will be given here.

(1) To measure the temperature in the inside of the black hole. If we admit that temperature is proportional to the mean translational kinetic energy of all particles including photons, one can emit a light signal into the black hole, and measure the change of light speed. The well-known result is that at the singularity of the black hole the speed of light is zero. Thus we have: $\left(T_{s}=0\right), T_{s}<T_{k}$. This conclusion has been proved above.

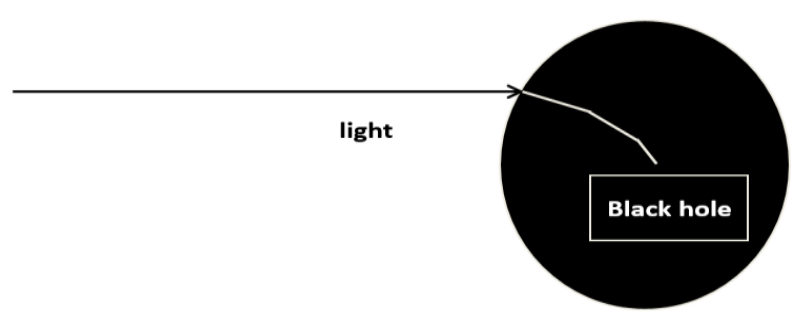

Fig 5. Schematic of the change in the velocity of light entering the black hole.

(2) Let the electrons stop moving.
At present, the means of obtaining ultra-low temperatures on earth are basically based on the idea of "stopping atoms from moving". If we want to get lower temperatures, we have to start with the idea that stops electrons from moving. At present, slowing down a single electron is hard to do in the lab. However, it is possible to accelerate or decelerate some of the electrons in the electron sea. For example, the laser and plasma wakefield accelerating technology is to accelerate some of electrons in some phase and position by making use of the wakefield produced by the laser. The principle is shown in Fig. 6.

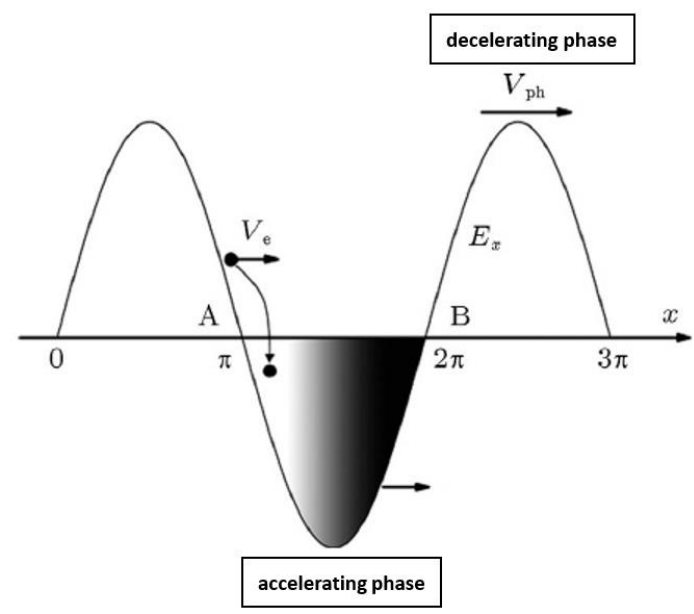

Fig 6. The principle of accelerating and decelerating some electrons in some phase and position.

From Fig.6 we can also see that there is a decelerating phase which means that these part of electrons can be decelerated. Evidence of electrons deceleration was first found in literature (Chou et al., 2016).

But how to decelerate the electrons at absolute zero has not been studied yet. A breakthrough in the future could make it possible for electrons to slow down step by step and eventually stop moving. If we define temperature by the velocity of electrons:

$$
\frac{1}{2} m \sigma_{e}^{2}=\frac{1}{2} k_{B} T
$$

where $\sigma_{e}$ is the root-mean-velocity of electrons, $k_{B}$ is Boltzmann constant, $T$ is temperature. When $\sigma_{e}$ equals zero, $T$ becomes zero, which is new absolute zero.

(3) There is a new absolute zero in Casimir vacuum.

In my previous paper $(\mathrm{Xu}, 2019)$ I proved that there is a new absolute zero temperature in Casimir vacuum.

\section{CONCLUSION}

Like any basic concept of science, the understanding of the nature of heat as well as temperature has a historical evolution. That the "phlogiston theory" and the "caloric theory" were replaced by the "motion theory of heat" is a great progress in history. However, this kind of motion is just limited to the molecular and atomic level, which doesn't seem to be enough now. Since heat is a kind of energy, and there is zero- point energy at absolute zero temperature, it means that there is still a temperature. This paper gives out the new definition of 
temperature: temperature is the degree of matter' motion. In this sense, this paper carefully examines the temperature inside the black hole, and find out that the electrons as well as other subatomic particles stop moving in the singularity of the black hole. The strong gravity will pull these electrons into their nuclei layer by layer (energy level). This is a cooling process. During this process, the energy will emit away, and the temperature will become lower and lower. Eventually the temperature will arrive at a new absolute zero. The existence

\section{REFERENCES}

Angeli, István, \& Marinova, Krassimira Petrova. (2013).

Table of experimental nuclear ground state charge radii: An update. Atomic Data and Nuclear Data Tables, 99(1), 6995.

Best, Nicholas W. (2015). Lavoisier's "Reflections on phlogiston" I: against phlogiston theory. Foundations of Chemistry, 17(2), 137-151. doi: 10.1007/s10698-015-92205

Carnot, NLS. (1897). Reflections on the Motive Power of Heat, edited by RH Thurston: Wiley.

Chandrasekhar, Subrahmanyan. (1984). On stars, their evolution and their stability. Reviews of modern physics, 56(2), 137.

Chou, Shao-wei, Xu, Jia, Khrennikov, K, Cardenas, Daniel E, Wenz, J, Heigoldt, M, . . . Karsch, Stefan. (2016). Collective deceleration of laser-driven electron bunches. Physical review letters, 117(14), 144801.

Fischer, J, Gerasimov, S, Hill, KD, Machin, G, Moldover, MR, Pitre, L, . . Ugur, H. (2007). Preparative steps towards the new definition of the kelvin in terms of the Boltzmann constant. International journal of thermophysics, 28(6), 1753-1765.

Fowler, Ralph H. (1926). On dense matter. Monthly Notices of the Royal Astronomical Society, 87, 114-122. of the new absolute zero can be proved by three ways: measuring the temperature at the singularity of the black hole, letting the electrons stop moving, and designing a Casimir vacuum pump. The finding of new absolute zero will be of great significance to the utilization of zero-point energy. Further works are needed to apply the results of this paper to the research of solving the puzzle of the singularity of the black hole, dark energy, cosmic models etc

Maxwell, James Clerk, \& Pesic, Peter. (2001). Theory of heat: Courier Corporation.

Medina, Sergio Bravo, Nowakowski, Marek, \& Batic, Davide. (2019). Einstein-Cartan Cosmologies. Annals of Physics, 400, 64-108.

Pacey, AJ, \& Fisher, SJ. (1967). Daniel Bernoulli and the vis viva of compressed air. The British journal for the history of science, 3(4), 388-392.

Purcell, Edward M, \& Pound, Robert V. (1951). A nuclear spin system at negative temperature. Physical Review, 81(2), 279.

Quan, Haitao T. (2009). Quantum thermodynamic cycles and quantum heat engines. II. Physical Review E, 79(4), 041129.

Rumford, Benjamin Count Of. (1798). An inquiry concerning the source of the heat which is excited by friction. by benjamin count of rumford, frsmria. RSPT, 88, 80-102. doi:https://doi.org/10.1098/rstl.1798.0006

Scovil, Henry ED, \& Schulz-DuBois, Erich O. (1959). Threelevel masers as heat engines. Physical Review Letters, 2(6), 262.

Slater, John C. (1964). Atomic radii in crystals. The Journal of Chemical Physics, 41(10), 3199-3204.

Xu, Xingwu. (2019). The Nature of Force and the Dark Matter. International Journal of Fundamental Physical Sciences (IJFPS), 9(3), 41-47. 